University of Wollongong

Research Online

Australian Institute for Innovative Materials -

Papers

Australian Institute for Innovative Materials

$1-1-2020$

\title{
Thermally actuated hydrogel bead based braided artificial muscle
}

Bidita Binte Salahuddin

bbs622@uowmail.edu.au

Holly Warren

University of Wollongong, hwarren@uow.edu.au

Geoffrey M. Spinks

University of Wollongong, gspinks@uow.edu.au

Follow this and additional works at: https://ro.uow.edu.au/aiimpapers

Part of the Engineering Commons, and the Physical Sciences and Mathematics Commons

Research Online is the open access institutional repository for the University of Wollongong. For further information contact the UOW Library: research-pubs@uow.edu.au 


\title{
Thermally actuated hydrogel bead based braided artificial muscle
}

\begin{abstract}
(c) 2020 IOP Publishing Ltd. Braided (McKibben) artificial muscles are one of the most attractive biomimetic actuators since they exhibit similar static and dynamic performance to skeletal muscles. One disadvantage of these systems, when intended for portable applications, is the need for a compressor or pump to provide pressurised fluid for muscle activation. An alternative is to use an expandable material, such as a hydrogel. This work investigates the development of hydrogel-based braided artificial muscles which can produce mechanical work through controlled hydrogel swelling in the presence of water. Hydrogel bead-filled braided meshes were prepared where no internal bladder was needed; since the bead size was larger than the holes in the braided mesh. A spring test method was introduced to measure force generation and strain developed by the braided mesh when the thermo-responsive gel was cooled from $60^{\circ} \mathrm{C}$ to $5{ }^{\circ} \mathrm{C}-8{ }^{\circ} \mathrm{C}$. Reducing the temperature resulted in hydrogel swelling and braided mesh length contraction due to the internal pressure generated. Blocked forces and actuation free strokes of 5-6 N and $7 \%-8 \%$ were observed, respectively. A long response time was the limiting factor of these actuators due to the slow diffusion properties of the hydrogel.
\end{abstract}

\section{Disciplines}

Engineering | Physical Sciences and Mathematics

\section{Publication Details}

Salahuddin, B., Hunt, H. \& Spinks, G. (2020). Thermally actuated hydrogel bead based braided artificial muscle. Smart Materials and Structures, 29 (5), 


\title{
Thermally actuated hydrogel bead based braided artificial muscle
}

\author{
Bidita Salahuddin ${ }^{1}$, Holly Warren ${ }^{1}$ and Geoffrey M. Spinks ${ }^{1 *}$ \\ ${ }^{1}$ ARC Centre of Excellence for Electromaterials Science and Intelligent Polymer Research Institute, \\ University of Wollongong, Innovation Campus, Squires Way, North Wollongong, NSW, 2522, Australia \\ *E-mail: gspinks@uow.edu.au
}

Received xxxxxx

Accepted for publication $\mathrm{xxxxx \textrm {x }}$

Published xxxxxx

\begin{abstract}
Braided (McKibben) artificial muscles are one of the most attractive biomimetic actuators since they exhibit similar static and dynamic performance to skeletal muscles. One disadvantage of these systems, when intended for portable applications, is the need for a compressor or pump to provide pressurised fluid for muscle activation. An alternative is to use an expandable material, such as a hydrogel. This work investigates the development of hydrogel-based braided artificial muscles which can produce mechanical work through controlled hydrogel swelling in the presence of water. Hydrogel bead-filled braided meshes were prepared where no internal bladder was needed; since the bead size was larger than the holes in the braided mesh. A spring test method was introduced to measure force generation and strain developed by the braided mesh when the thermo-responsive gel was cooled from $60^{\circ} \mathrm{C}$ to $5-8^{\circ} \mathrm{C}$. Reducing the temperature resulted in hydrogel swelling and braided mesh length contraction due to the internal pressure generated. Blocked forces and actuation free strokes of 5-6 $\mathrm{N}$ and 7-8\% were observed, respectively. A long response time was the limiting factor of these actuators due to the slow diffusion properties of the hydrogel.
\end{abstract}

Keywords: artificial muscle, braided actuator, hydrogel bead, actuation stroke

\section{Introduction}

Artificial muscles are devices or materials which can reversibly contract, expand, or rotate when acted upon by an external stimulus such as electricity, $\mathrm{pH}$, pressure, magnetic field or temperature [1] . In recent years, significant advances have been made in artificial muscles and their potential to make sophisticated biologically inspired systems [2-6] . This technology has many prospective future applications in industry, medicine, robotics and many other fields [3, 7-10].

McKibben muscles are a popular type of artificial muscle due to their similarity in performance to biological muscles as well as their high actuation force per mass [11-13]. These systems use an inflatable bladder that operates against an external braid such that pressurisation of the bladder causes a contraction in braid length. Typically, air pressure is used to make pneumatic artificial muscle (PAMs). Alternatively, hydrogen gas pressure in the metal hydrate reactors can also be used to drive the PAMs $[14,15]$.

Recently, hydraulic McKibben muscles (HAMs) have been introduced as a new generation of these muscles operated by pressurised water or oil [16-19]. Both of these muscles display lightweight, high force capability, low static friction, and compliance. Nonetheless, these artificial muscles show some disadvantages, for instance, the requirement of a separate mechanical air compressor or fluid pump, a noisy system due to exhaust during depressurisation, and a heavy system to carry for portable applications such as wearable systems or mobile robots [20]. Several attempts have been made to replace the fluid pressure with chemo-sensitive materials to overcome those disadvantages. For example, a new type of 
McKibben muscle has been introduced by replacing air with $\mathrm{pH}$-sensitive hydrogel spheres with a pump alternatively delivering acidic or basic solutions to cause gel swelling and de-swelling. These muscles offer reasonable performances; however, they suffer from a long response time (>30 min) [21] and still require an external pump. Another attempt has been made with braids filled with dry hydrogel powder or cylinders, where immersion in water was used for gel swelling [22] . These muscles generated useful forces and displacements, but with problems of reversibility and repeatability.

In this work, the use of a thermally-responsive hydrogel is investigated as a means for reversibly controlling the pressure applied to a braided muscle. These gel-filled muscles (GFMs) were tested by immersing in hot and cold water baths with temperatures above and below the hydrogel's lower critical solution temperature (LCST) of $32-34^{\circ} \mathrm{C}$. A customdeveloped test method is also proposed where the gel beadfilled muscle was loaded against an extension spring in series. This simple method enabled the determination of both the blocked force and free stroke of the muscle as a result of gel swelling. Alginate/PNIPAAm ionic-covalent entanglement (ICE) hydrogel beads were synthesised to use in the muscles by incrementally varying the NIPAAm concentration from 1.5 to $2.5 \mathrm{w} / \mathrm{v} \%$.

\section{Experimental}

\subsection{Materials}

$\mathrm{N}, \mathrm{N}$ '-bismethylene acrylamide (MBAA) crosslinker, alginic acid sodium salt (SA), calcium chloride $\left(\mathrm{CaCl}_{2}\right)$, and $\mathrm{N}, \mathrm{N}, \mathrm{N}^{\prime}, \mathrm{N}^{\prime}$-tetramethylethylene diamine (TEMED) were purchased from Sigma Aldrich, Australia. Similar grade Nisopropylacrylamide (NIPAAm) were obtained from two different sources, Sigma Aldrich, Australia and Wako Pure Chemical Industries Ltd., China. Ammonium persulfate (APS) was supplied by Ajax Fine Chem, Australia. Cylindrical braids (poly phenylene sulphide, PPS) were purchased from JDD TECH Company, China. All materials were used as-received, and all solutions were prepared using Milli-Q water (resistivity $=18.2 \Omega \mathrm{cm}$ ).

\subsection{Preparation of hydrogel bead}

Hydrogel beads were prepared from ICE gels, using ionically crosslinked alginate with an interpenetrating PNIPAAm covalent network, as follows. Firstly, calcium alginate beads were synthesized by a dripping method; drops of alginate solution (1.9 wt\% in water) were dripped through a nozzle of hand-held airbrush (Aztek A4709) into an aqueous $\mathrm{CaCl}_{2}$ solution $(1.1 \mathrm{wt} \%)$. These parameters were chosen following a series of optimisation experiments using different chemical compositions for preparing the ICE hydrogel beads in terms of their swelling capability and Young's modulus in both the hot and cold environments. The attained calcium alginate beads were left to stand in the $\mathrm{CaCl}_{2}$ solution for 24 h. Next, the calcium alginate beads were immersed in $10 \mathrm{~mL}$ of a buffer solution containing NIPAAm monomer (1.5-2.5 wt \%), N, N'-methylenebisacrylamide (MBAA,crosslinker, 0.2-0.6 wt $\%$ ), ammonium persulfate (APS, initiator, $0.1 \mathrm{wt} \%$ ) and $\mathrm{CaCl}_{2}(1.1 \mathrm{wt} \%)$. The solution was then degassed for 1 hour at $0^{\circ} \mathrm{C}$ using $\mathrm{N} 2$. Later, the calcium alginate beads were collected, excess surface water was removed through wiping, and the beads were redispersed in cyclohexane $(10 \mathrm{~mL})$. By adding tetramethylethylenediamine (TEMED) $(300 \mu \mathrm{L} \mathrm{10 \%}$ $\mathrm{v} / \mathrm{v}$ solution) to the cyclohexane, polymerization was initiated inside the beads and continued for 24 hours at $4{ }^{\circ} \mathrm{C}$. The beads were separated after polymerization from the cyclohexane and washed several times with water. Finally, the beads were stored in water at $4^{\circ} \mathrm{C}$.

\subsection{Swelling}

The swelling ratio of the ICE hydrogels in water was determined at $22^{\circ} \mathrm{C}$ by measuring the change in weight. The hydrogels were initially immersed in water for 72 hours and the swelling ratios, $Q$ were calculated using:

$$
Q=\frac{W_{S}-W_{D}}{W_{D}} \times 100 \%
$$

Here, $w_{D}$ is the weight of dry hydrogel and $w_{S}$ is the weight of swollen hydrogel. Samples were dried by placing under a fume hood at $22^{\circ} \mathrm{C}$ for 72 hours.

\subsection{Thermo-responsive gel actuation}

The actuation of ICE hydrogels in water was stimulated by cycling the temperature between $5^{\circ} \mathrm{C}$ and $60^{\circ} \mathrm{C}$. The ICE hydrogels were immersed in water at $60^{\circ} \mathrm{C}$ and then at $5^{\circ} \mathrm{C}$ for 1 hour each. The swelling ratio of the PNIPAAm hydrogels in water was determined during actuation by measuring the change in volume using a macroscope (Leica Macroscope Z16, Leica Microsystems, Germany) in tandem with Leica Application Suite software. The volumetric swelling ratios, $V$, were calculated using:

$$
V=\frac{V_{c o l d}-V_{h o t}}{V_{h o t}} \times 100 \%
$$

Here, $V$ is the volumetric swelling ratio of a bead with respect to the initial volume at $60^{\circ} \mathrm{C}\left(v_{\text {hot }}\right)$ where $v_{\text {cold }}$ is the volume at $5^{\circ} \mathrm{C}$.

\subsection{Fabrication of Gel Filled Muscle}

Figure 1 shows the fabrication process of gel bead filled braided muscle. First, $631 \mathrm{mg}$ of dried hydrogel beads were 


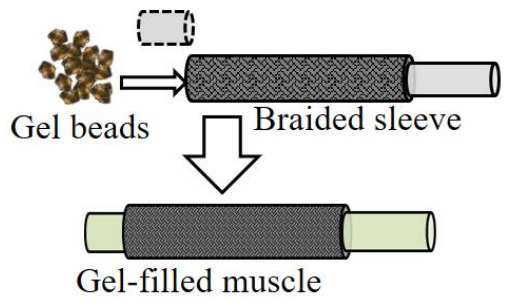

(a)

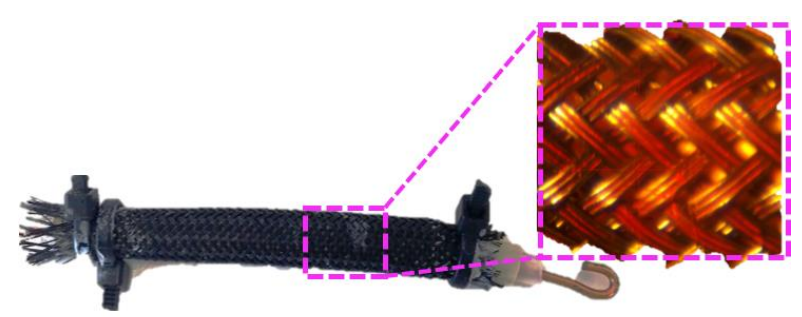

(b)

Figure 1. (a) Schematic illustration of the fabrication of gel-filled muscle. (b) An actual photograph of as-fabricated muscle. Inset showing a micrograph of the braid surface.

inserted into a braided sleeve (polyphenylene sulfide, PPS), and both ends of the muscle were blocked to prevent bead escape when the muscle was immersed in a water bath. The initial, unloaded length and diameter of the muscles equilibrated in $60^{\circ} \mathrm{C}$ water were 47 and $6.5 \mathrm{~mm}$, respectively.

\subsection{Experimental set-up for Gel Filled Muscle}

An in-house experimental set-up was designed to measure force, displacement and stiffness of gel-filled muscle. A beaker $(250 \mathrm{~mL})$, the gel bead-filled muscle, a thermocouple and a laser displacement detector were used together, as in figure 2. An e-Corder data logger (ED 410, e-DAQ) was used to process the signals from the laser detector and thermocouple. A range of spring lengths (11 to $55 \mathrm{~mm}$ ) with different spring constants (102 to $1020 \mathrm{~N} / \mathrm{m}$ ) was used to conduct the tests. One end of the spring was firmly fixed to a rigid support of the test set-up while other end was attached to the gel bead filled muscle in series.

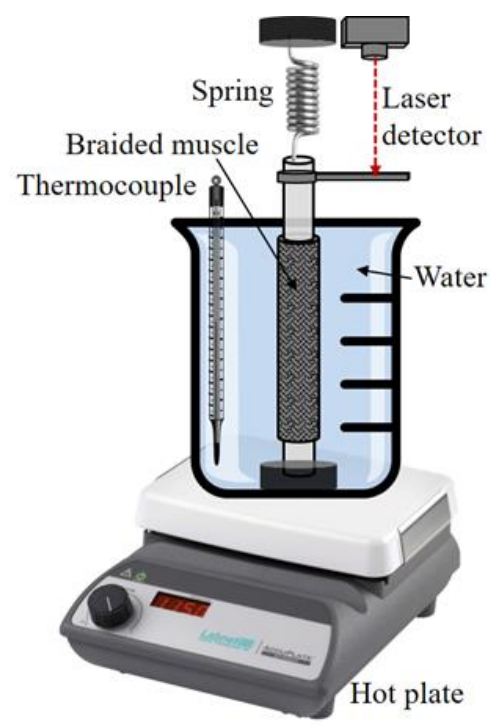

Figure 2. Schematic illustration of the actuation test set-up developed in-house.

A hot plate and ice were used to maintain the temperature of the water bath at the hot and cold states, respectively. The gel-filled muscle was attached to a spring and immersed in hot water at $60^{\circ} \mathrm{C}$, and then the hot water was exchanged for cold water of $8^{\circ} \mathrm{C}$ for 2 hours duration. During this period, the length change was recorded.

Free strain obtained from the experiments was utilized to calculate the relevant force generation from Hooke's law [23]:

$$
F=k X
$$

Here, $k$ is a constant characteristic factor of the spring related to its stiffness and $X$ is the muscle displacement which was kept comparatively small compared to the total possible deformation of the spring. Spring stiffness, $k$ was measured by using Hooke's Law [23]:

$$
F_{S}=k x_{s}
$$

Here, $F_{S}$ is the required force to extend the spring and $x_{s}$ is the extension of the spring. For this experiment, one side of the spring was fixed to the clamp while the other side attached to the weight holder. Upon increasing weight load, the extension of the spring was found from the data logger (ED 410 , e-DAQ) software. Eventually, $k$ is measured by the slope of the force (applied load) vs spring extension curve.

\section{Results and discussion}

\subsection{Morphology}

Figure 3 shows microscopic images of a typical ICE hydrogel beads ( $2.5 \%$ NIPAAm concentration) at $22^{\circ} \mathrm{C}, 60^{\circ} \mathrm{C}$ and $5^{\circ} \mathrm{C}$. Comparison of the hydrogel beads from dry to the swollen state in the water at $22^{\circ} \mathrm{C}$ shows that the gel volume expanded by the water swelling at this temperature, which is well below the PNIPAAm phase transition temperature [24]. Heating in a $60^{\circ} \mathrm{C}$ water bath caused the gel to de-swell significantly, although the volume is still much larger than in the dry state. The optical view of the hydrogel in the swollen state below the transition temperature $\left(22^{\circ} \mathrm{C}\right.$ and $\left.5^{\circ} \mathrm{C}\right)$ is transparent and opaque when de-swollen at $60^{\circ} \mathrm{C}$.

The Amount of hydrogel swelling by absorption of water is determined by a balance of the osmotic forces that extend the polymer network and the opposing elastic forces obtained from the stretched polymer segments. To examine the potential for thermally driven actuation, the hydrogels were equilibrated at different temperatures of $60^{\circ} \mathrm{C}$ and $5^{\circ} \mathrm{C}$ which 

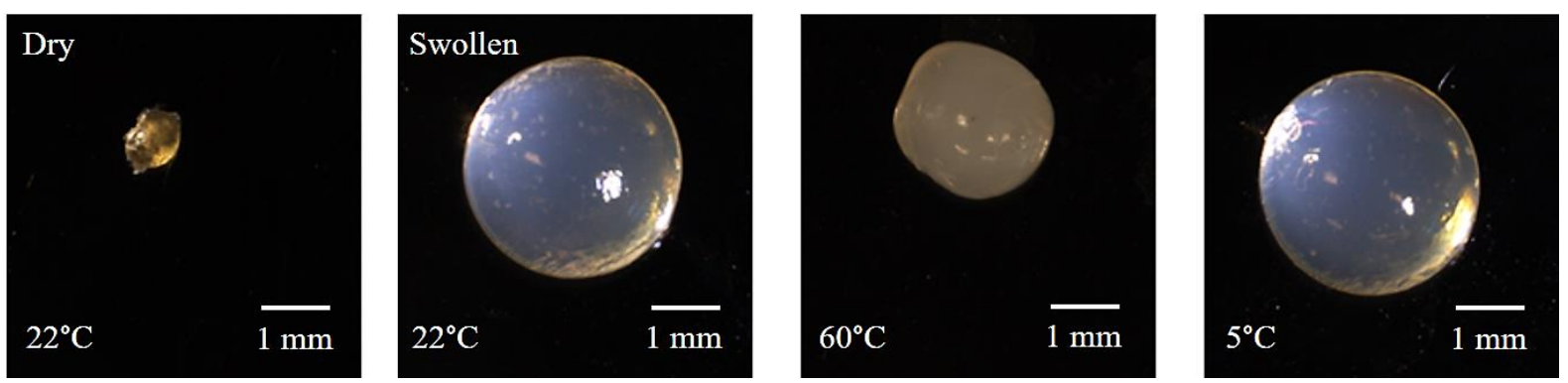

Figure 3. Microscopic images of a typical ICE hydrogel bead in the dry state and when swollen in the water at $22^{\circ} \mathrm{C}, 60^{\circ} \mathrm{C}$ and $5^{\circ} \mathrm{C}$.

were well above and well below the transition region of 32$34^{\circ} \mathrm{C}$. Reversible swelling and de-swelling behaviour were confirmed in the ICE hydrogels prepared in this study. In all cases, the diameter of the hydrogels, when equilibrated at temperatures below $\left(22^{\circ} \mathrm{C}\right.$ or $\left.5^{\circ} \mathrm{C}\right)$ the LCST of PNIPAAm, was larger than that of the diameter of the same gels when equilibrated at $60^{\circ} \mathrm{C}$, as depicted in figure 3 . The optical colour of the hydrogel was also changed from opaque to transparent as temperature changes from above to below the LCST. At the temperatures above the LCST, the beads become opaque by the squeezing out of the water molecules from the hydrogel which causes an internal density inhomogeneity which scatters visible light [25].

\subsection{Characteristic properties of ionic- covalent entanglement hydrogel}

In order to examine the actuation of hydrogel bead, the swelling ratio was monitored as a function of NIPAAm concentration at different temperatures. Figure 4 shows the swelling ratio of ICE hydrogels at different temperature conditions. Figure 4(a) shows that the weight-based swelling ratio of ICE hydrogel when equilibrated in room temperature water and when prepared at different PNIPAAm concentrations of $1.5,1.7,2.1$, and $2.5 \mathrm{w} / \mathrm{v} \%$. The gel swelling ratio increased with the increase of NIPAAm concentration used to prepare the ICE hydrogels. The increasing NIPAAm concentration leads to the increased swelling ratio of ICE hydrogel when these gels were equilibrated in the water below the LCST. Typically, the equilibrium swelling ratio of the hydrogel is controlled by the crosslink density and that the crosslink density of hydrogel is determined by the molar ratio of PNIPAAm monomer to MBAA crosslinker [26]. The
MBAA crosslinker concentration was kept constant in the monomer solution so that the ratio of NIPAAm monomer to MBAA crosslinker increased as the concentration of NIPAAm increased. A lower crosslink density is expected as the monomer to crosslinker ratio increased.

To evaluate the NIPAAm concentration of ICE hydrogels for thermally induced actuation, swelling ratios were also determined at temperatures well above $\left(60^{\circ} \mathrm{C}\right)$ and well below $\left(5^{\circ} \mathrm{C}\right)$ the swelling transition temperature. The swelling was obtained for each of the ICE hydrogels, as shown in figure 4(b). The swelling ratio was determined by measuring the volume change of the hydrogel at $5^{\circ} \mathrm{C}$ based on the minimum gel volume at $60^{\circ} \mathrm{C}$. An opposite trend of the swelling ratio with NIPAAm concentration was found for these hydrogels at the two temperatures. At $60^{\circ} \mathrm{C}$, the volumetric swelling ratio decreased with increasing NIPAAm concentration; while at $5^{\circ} \mathrm{C}$, the swelling ratio increased with increasing NIPAAm concentration. This could be possible due to the degree of swelling in the hydrogel is controlled by the increased amount of NIPAAm concentration in the crosslinked network leading to enhanced water uptake triggered by the temperature sensitivity of NIPAAm at lower LCST $\left(5^{\circ} \mathrm{C}\right)$. The decreasing swelling ratio at $60^{\circ} \mathrm{C}$ was caused by the high thermoresponsive characteristic of the highly concentrated NIPAAm gel. As the preferred temperature for the actuation was higher than the PNIPAAm thermal transition, therefore hydrogel was speedily heated and generated rapid actuation at higher NIPAAm content. Since the hydrogel filled braided muscles will be stimulated by changing the temperature through the transition region, the hydrogels prepared with different PNIPAAm concentrations are likely to show different actuation behaviour as a result of the significant changes in swelling, as shown in figure 4(b). 

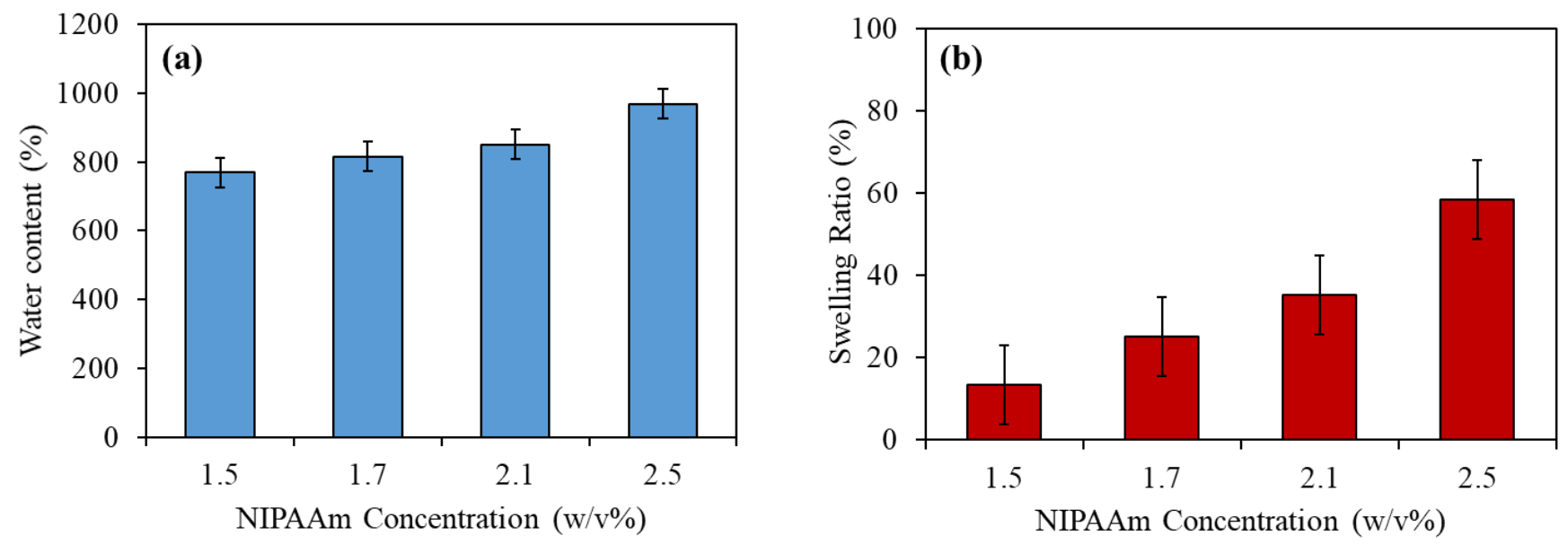

Figure 4. (a) The weight-based water content of ICE hydrogel beads at varying PNIPAAm concentrations and after equilibrating in the water at $22^{\circ} \mathrm{C}$. (b) The volumetric swelling ratios of thermally induced ICE hydrogel beads with different NIPAAm concentration and when cooled from $60^{\circ} \mathrm{C}$ to $5^{\circ} \mathrm{C}$.

Figure 5 illustrates Young's moduli of the ICE hydrogels prepared with different NIPAAm concentrations and tested after equilibrating in the water at $60^{\circ} \mathrm{C}$ and $5^{\circ} \mathrm{C}$. As shown in the figure, the increasing NIPAAm concentration caused an increase in Young's modulus of the hydrogel when equilibrated and tested at $60^{\circ} \mathrm{C}$.

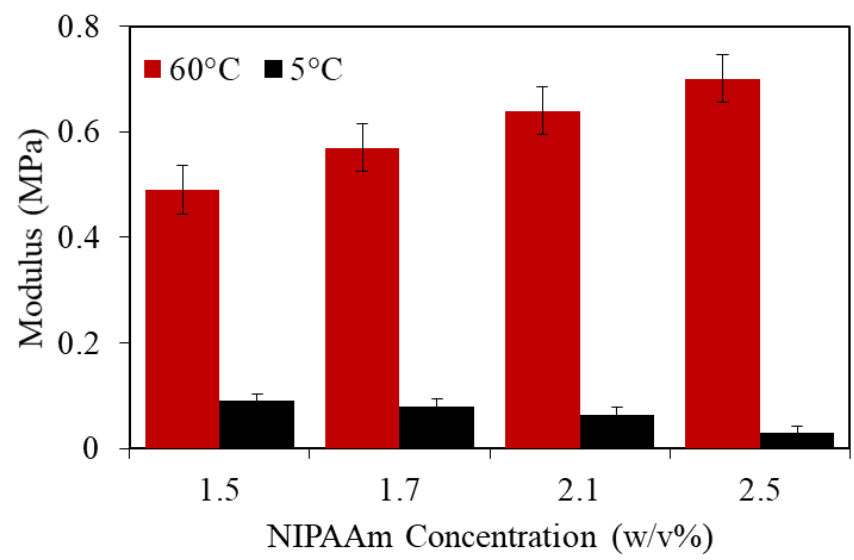

Figure 5. Average Young's modulus of ICE hydrogel obtained at $60^{\circ} \mathrm{C}$ and $5^{\circ} \mathrm{C}$ having NIPAAm concentration of $1.5,1.7,2.1$, and 2.5 $\mathrm{w} / \mathrm{v} \%$.

In contrast, there was a decreasing trend in Young's modulus with increasing PNIPAAm concentration when the hydrogels were equilibrated and tested at $5^{\circ} \mathrm{C}$. Because the modulus of hydrogels is also determined by the crosslink density and degree of swelling as the modulus is determined by the concentration of network chains [25, 27]. With increasing NIPAAm monomer, the crosslink density of the hydrogels decreased, which corresponds to a decreasing Young's modulus for hydrogels below LCST. For the hydrogels above LCST, the modulus was strongly affected by the degree of swelling with samples of a higher NIPAAm concentration having a higher modulus due to their reduced swelling. For further experimental observation, 2.5 wt.\% NIPAAm was chosen to verify the theoretically calculated blocked force as a function of time due to these findings.

\subsection{Actuation results of gel filled muscle}

Time based actuation response of artificial muscles is important to evaluate the dynamic output that a muscle can demonstrate. Figure 6 illustrates the actuation test results of four different GFMs prepared using ICE gel beads with varying NIPAAm monomer concentrations and with the test conducted by using six springs with different spring constants (stiffness of the springs decreased in numerical order with Spring 6 being the stiffest and Spring 1 being the least stiff). Table 1 shows the length of the springs used together with relevant spring constants.

Table 1 Dimension and the stiffnesses of the springs.

\begin{tabular}{|l|l|l|}
\hline Spring identification & $\begin{array}{l}\text { Spring length } \\
(\mathrm{mm})\end{array}$ & $\begin{array}{l}\text { Spring constant } \\
(\mathrm{N} / \mathrm{m})\end{array}$ \\
\hline 1 & 11 & 102 \\
\hline 2 & 16 & 220 \\
\hline 3 & 23 & 375 \\
\hline 4 & 29 & 565 \\
\hline 5 & 34 & 712 \\
\hline 6 & 55 & 1020 \\
\hline
\end{tabular}

In all cases, it was observed that the higher the spring constant, the lower was the displacement of the muscle when the gel volume transition was stimulated by decreasing the water bath temperature from above to below the LCST. Moreover, it was also observed that GFMs prepared with higher NIPAAm concentrations exhibited a greater displacement when operated against a given spring. Overall, the slow response time was apparent for GFM in each case, demonstrating a limitation of muscle feasibility in certain practical applications. 

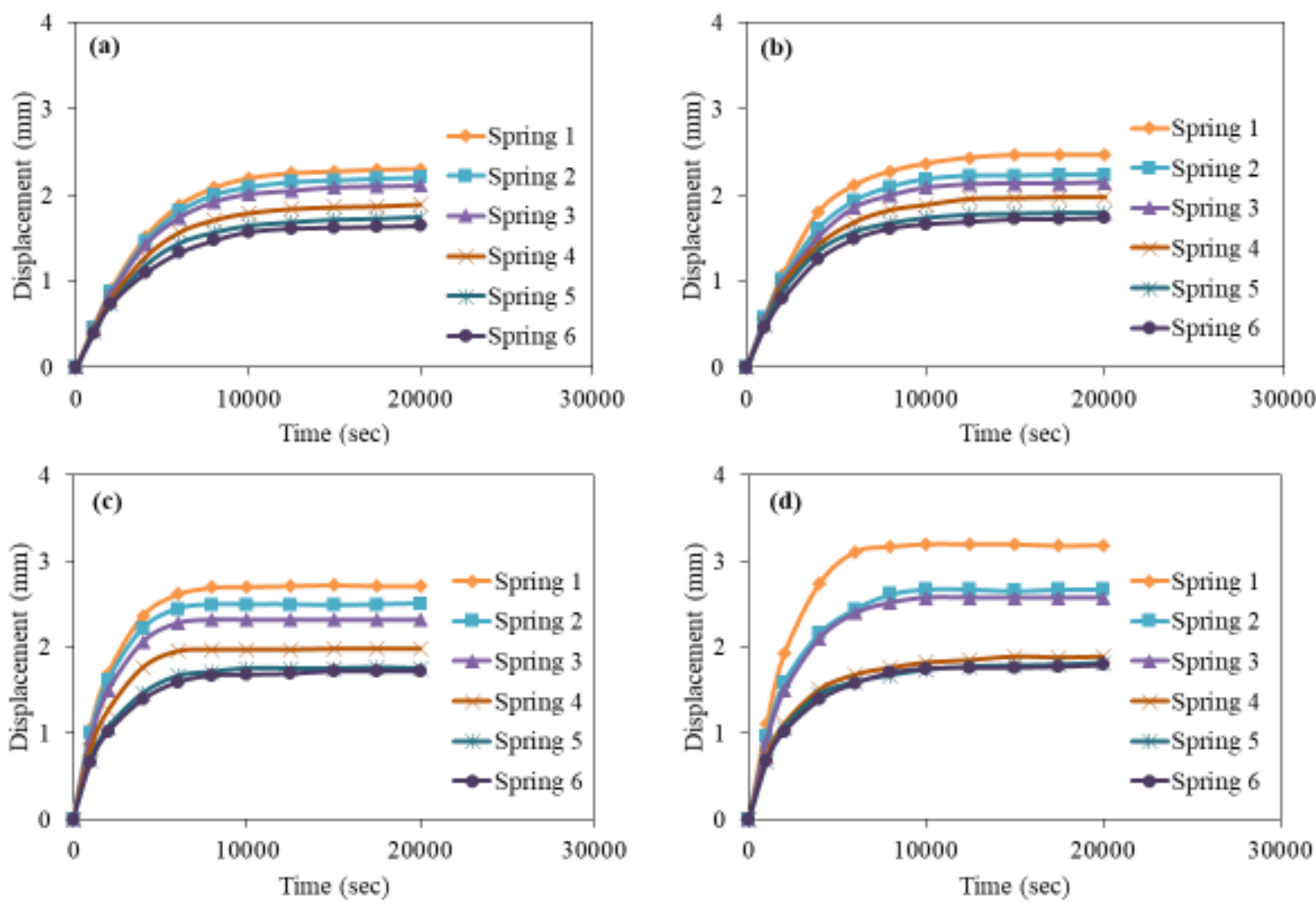

Figure 6. Contractile length displacement during cooling from $60^{\circ} \mathrm{C}$ to $8^{\circ} \mathrm{C}$ as a function of time for GFMs having unloaded length of $47 \mathrm{~mm}$ each tested against 6 different springs. A series of GFMs were investigated that were prepared with different NIPAAm compositions of (a) $1.5 \mathrm{w} / \mathrm{v} \%$, (b) $1.7 \mathrm{w} / \mathrm{v} \%$, (c) $2.1 \mathrm{w} / \mathrm{v} \%$, and (d) $2.5 \mathrm{w} / \mathrm{v} \%$. All samples were first equilibrated in a water bath at $60^{\circ} \mathrm{C}$ and then immersed in a water bath at $8^{\circ} \mathrm{C}$, at time $=0$ seconds.

Figure 7(a) illustrates the force (calculated from equation 2.3) as a function of stroke for gel-bead filled GFM fabricated using different NIPAAm concentrations and each tested with six different springs. A linear relationship was seen for each gel type when the results were plotted as the force generated versus displacement. These results were extrapolated to both force and displacement axes to predict the amount of blocked force and free stroke that can be generated by each muscle. Again, it was confirmed that increasing the amount of NIPAAm in the gel beads generated more force and more displacement. At the maximum NIPAAm concentration, the GFM generated an estimated blocked force of $5.1 \mathrm{~N}$, which corresponded to blocked stress of $0.04 \mathrm{MPa}$ when normalised to the unloaded cross-sectional area of the muscle. A separate experimental observation was made using a mechanical tester to verify the blocked force that was theoretically calculated from the data in figure 7(a). Figure 7(b) shows 2 cool/heat cycles of isometric force generation as a function of time from gel-bead filled GFM containing 2.5 w/v\% NIPAAm concentration. As shown in the first cycle, fully reversible force generation with $\sim 5.14 \mathrm{~N}$ blocked force was obtained which shows very good agreement with the calculated value of $\sim 5.1 \mathrm{~N}$ obtained by extrapolation of the spring data. The second cycle was conducted in a 3 -step process where cold water was introduced in the first step, then replaced by room temperature water, and finally changed to hot water. This process also showed very similar force generation in cold condition but demonstrated significant stress relaxation at room temperature. Introducing hot water assisted the muscle to release all the generated force and concluded a fully reversible actuation cycle. It has been observed that the force generation at the cold temperature was much slower than the force release at a hot temperature. The swelling behaviour of the hydrogel is actively controlled by the diffusion of the water which is related to the relaxation of the polymer chains. At higher temperatures, the polymeric network of PNIPAAm contracts readily and actively squeezes to release the water. At lower temperature, the relaxation of the polymer chain takes a longer time to unfold and accommodate the swelling [28, 29].

The maximum free strain obtained from the GFMs was $7.66 \%$ based on the unloaded length. The free strain increased with increasing NIPAAm concentration used to prepare the ICE gel beads. This study has demonstrated that GFMs exhibit significant force generation capability and significant contractile actuation when cooled from above LCST to below LCST. The actuation was reversible through multiple cold/hot cycles. 

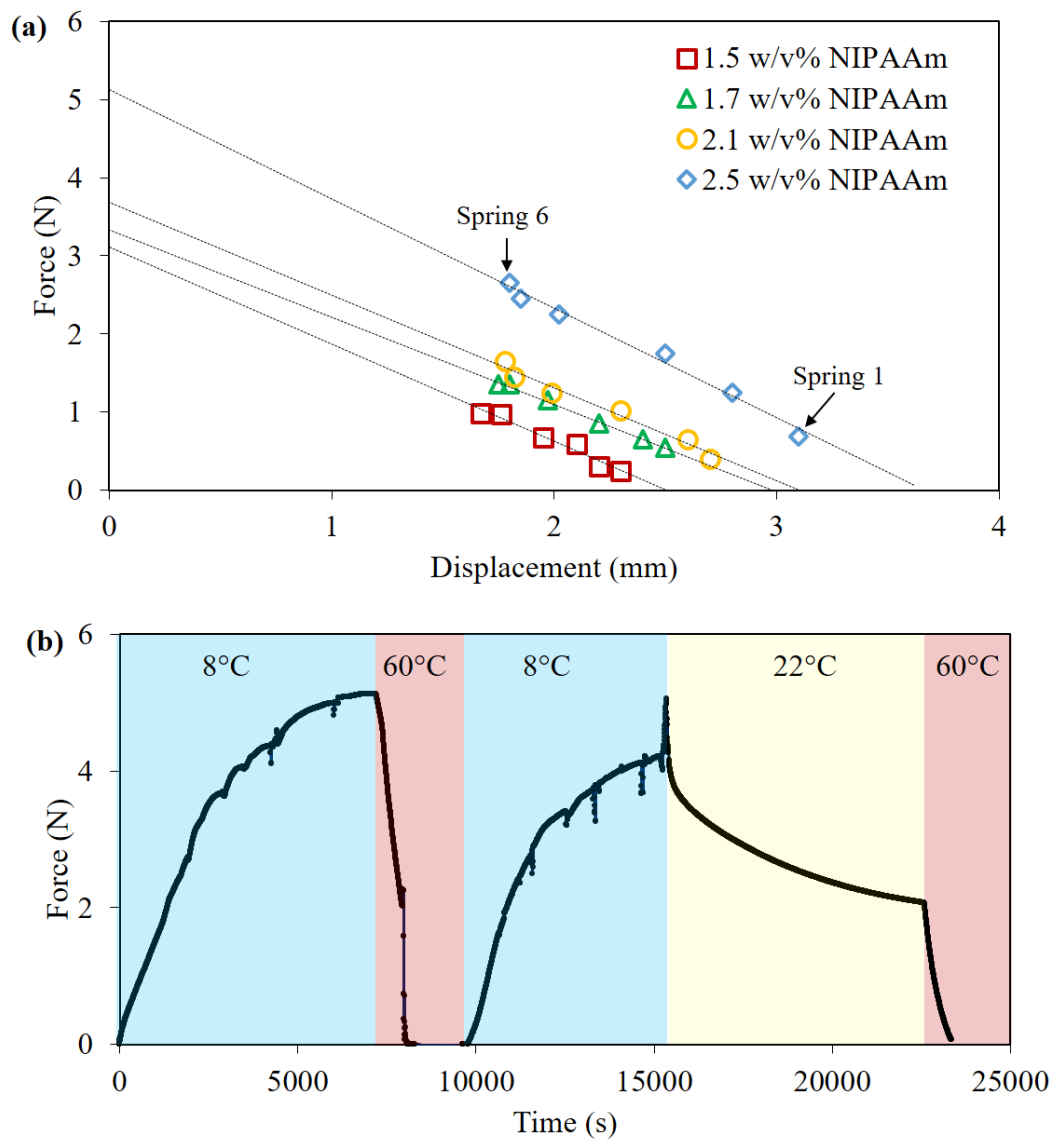

Figure 7. (a) Force-displacement curves for GFM with different NIPAAm compositions each tested with 6 different springs (each data point represents a particular spring). The slope of the dotted line represents the stiffness of the GFM at $8^{\circ} \mathrm{C}$ temperature. (b) Force generation hydrogel ( $2.5 \mathrm{w} / \mathrm{v} \%$ NIPAAm concentration) beads GFM as a function of time and when immersed in cold, hot, or normal water baths at the times indicated

The effect of GFM dimension on the actuation properties were also evaluated by using the muscles of different diameter ( $5 \mathrm{~mm}$ and $6 \mathrm{~mm}$ ) but keeping the length consistent $(47 \mathrm{~mm})$ to the previously used $6.5 \mathrm{~mm}$-diameter sample. The NIPAAm concentration was $2.5 \%$ in all cases and the amount of gel loading in the 5-mm and 6-mm diameter were 485 and $582 \mathrm{mg}$, respectively. The packing fraction of dry gel in the braids varied slightly from $0.53 \mathrm{mg} / \mathrm{mm}^{3}$ to $0.44 \mathrm{mg} / \mathrm{mm}^{3}$ to $0.41 \mathrm{mg} / \mathrm{mm}^{3}$ as the braid diameter increased from $5 \mathrm{~mm}$ to 6 $\mathrm{mm}$ to $6.5 \mathrm{~mm}$. The force-displacement results were produced against springs 1-6 and compared to that of similarly composed muscle having 6.5-mm diameter (Figure 8). The results clearly demonstrate that the ability of the different diameter GFMs to produce force and displacement increased with increasing muscle diameter.

This phenomenon can be better explained by considering the blocked stress normalised by the gel packing factor. Table 2 shows the parameters that contributed to the final blocked stress. Once the different gel contents were considered, there was little difference in the stress generated by the GFMs having different diameters.

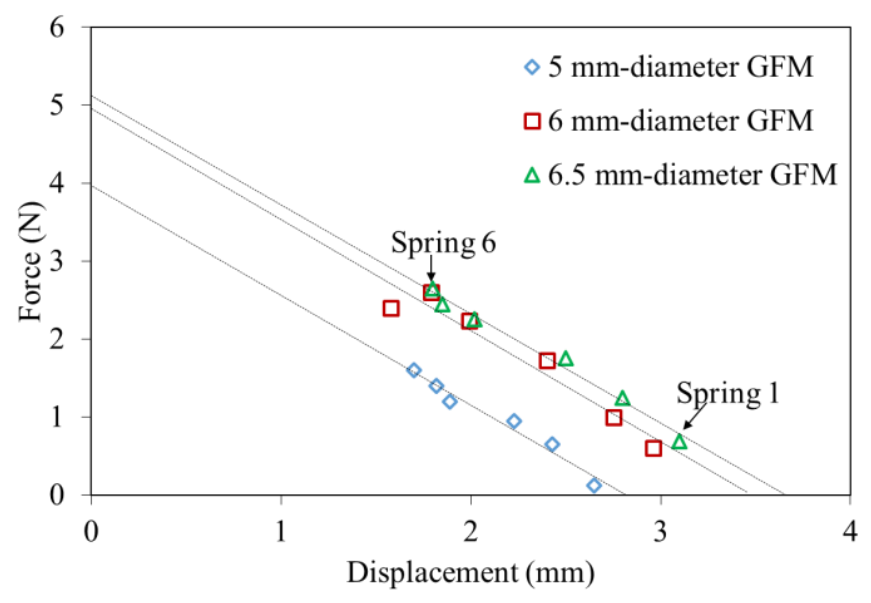

Figure 8. Force-displacement curves for GFM of different diameter each tested with 6 different springs. The slope of the dotted line represents the stiffness of the GFM at $8^{\circ} \mathrm{C}$ temperature. 
Table 2 Dimension and properties of GFMs prepared with different diameter braids.

\begin{tabular}{|l|l|l|l|l|l|l|}
\hline $\begin{array}{l}\text { Muscle } \\
\text { diameter }(\mathrm{mm})\end{array}$ & $\begin{array}{l}\text { Volume } \\
\left(\mathrm{mm}^{3}\right)\end{array}$ & $\begin{array}{l}\text { Gel mass } \\
(\mathrm{mg})\end{array}$ & $\begin{array}{l}\text { Packing factor } \\
\left(\mathrm{mg} / \mathrm{mm}^{3}\right)\end{array}$ & $\begin{array}{l}\text { Blocked force } \\
(\mathrm{N})\end{array}$ & $\begin{array}{l}\text { Blocked stress } \\
(\mathrm{MPa})\end{array}$ & $\begin{array}{l}\text { Blocked stress / Packing } \\
\text { factor }\left(\mathrm{MPa} . \mathrm{mm}^{3} / \mathrm{mg}\right)\end{array}$ \\
\hline 5 & 922.8 & 485 & 0.53 & 4 & 0.20 & 0.39 \\
\hline 6 & 1328.9 & 582 & 0.44 & 4.95 & 0.18 & 0.40 \\
\hline 6.5 & 1559.6 & 631 & 0.40 & 5.1 & 0.15 & 0.38 \\
\hline
\end{tabular}

\subsection{Theoretical analysis of actuation results}

Theoretical understanding of the blocked force generated from GFMs can be obtained by calculating the pressure generated by the swelling gel when the temperature changed from $60^{\circ} \mathrm{C}$ to $5^{\circ} \mathrm{C}$. This pressure is first calculated from the measured blocked force according to theory reviewed by Tondu [30] for McKibben muscles and based on the principle of virtual work. For an ideal cylindrical McKibben muscle, the general muscle force equation is [30]:

$$
\left.F_{\text {ideal }}=\left(\pi r_{0}^{2}\right) P\left[a(1-\varepsilon)^{2}-b\right)\right]
$$

Here, $r_{0}$ is the braid cylinder initial radius, $P$ is the applied pressure, $\varepsilon$ is the actuation strain, $\alpha_{0}$ is the initial braid angle, $a=3 / \tan ^{2} \alpha_{0}$ and $b=1 / \sin ^{2} \alpha_{0}$. The maximum or blocked force that the muscle will produce when $\varepsilon=0$, and the free strain is achieved when no force is generated. For these two cases, Eq. (5) can be deduced as:

$$
\begin{aligned}
& F_{\text {blocked }}=\left(\pi r_{0}^{2}\right) P(a-b) \\
& \varepsilon_{\text {free }}=1-\left[1 /\left(\sqrt{3} \cos \alpha_{0}\right)\right]
\end{aligned}
$$

Experimentally measured blocked forces were used to determine ideal pressures from Eq. (6) for the GFMs made of $1.5,1.7,2.1$, and $2.5 \mathrm{w} / \mathrm{v} \%$ NIPAAm concentrations. These calculations used initial diameter and braid angles at $60^{\circ} \mathrm{C}$ of $8.4 \mathrm{~mm}$ and $47.1^{\circ}$, respectively. These calculated pressures are shown in Figure 9 to increase approximately linearly with an increase in the NIPAAm concentration.

Additionally, the pressure expected due to the volume change of gel beads can be calculated from the bulk modulus $(B)$ of each type of gel beads and the change in gel volume $(\Delta V)$ relevant to the initial volume $\left(V_{0}\right)[31]$ :

$$
P=B \frac{\Delta V}{V_{0}}
$$

Here the fractional change in volume is for gel beads compressed at $5^{\circ} \mathrm{C}$ to their original $60^{\circ} \mathrm{C}$ diameter. $B=$ $E /[3(1-2 v)] ; E$ is Young's modulus and $v$ is the Poisson's ratio. Bulk moduli of the gels were calculated from corresponding Young's moduli in the final state at $5^{\circ} \mathrm{C}$, and a typical Poisson's ratio $(\sim 0.48)$ reported elsewhere for NIPAAm gels at temperatures lower than the LCST [32]. The pressures determined from the bead volume change and bulk moduli are also included in Figure 9. These calculated pressures show an apparent maximum pressure generated at a
PNIPAAm concentration of $2 \%(\mathrm{w} / \mathrm{v})$ which is in contrast to the steady increase in pressure with PNIPAAm concentration for pressures calculated from the blocked force. However, it should be noted that pressures calculated from the bulk moduli and swelling ratio are very sensitive to the Poisson's ratio and any trend shown in Figure 9 should be treated with caution. Despite this anomaly, it is clear that the absolute values of pressure obtained using both calculation methods are in reasonable agreement. It is, therefore, possible to make approximate predictions of GFM performance based on the swelling ratio and swollen-state modulus of the gel.

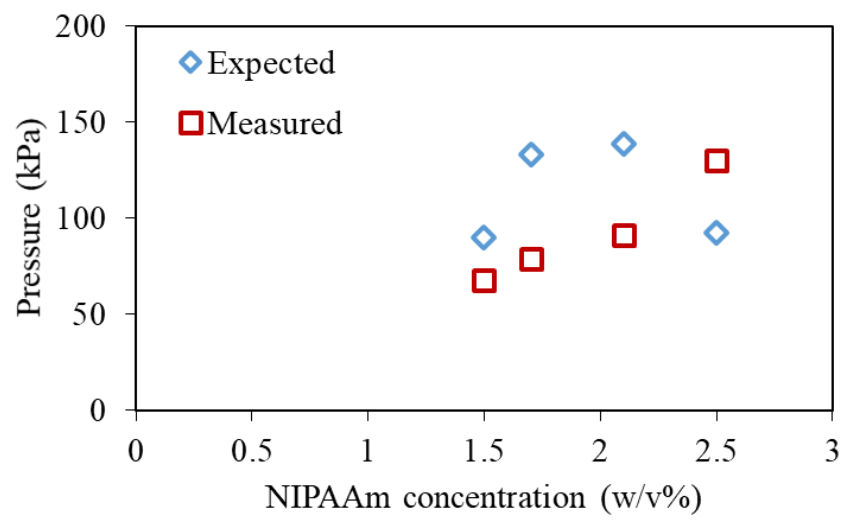

Figure 9. Comparison of calculated pressure results of GFMs in terms of different NIPAAm concentrations. The pressures were calculated for each GFM from both the measured blocked force (Measured) and the change in volume and moduli (Expected).

Actuation results obtained from GFM were further compared to the actuation performance that is ideally expected from a typical pneumatic muscle having a similar dimension. When subjected to a similar internal pressure (approx.130 $\mathrm{kPa}$ ) as generated by the actuated gel beads, the blocked force and free strain of the ideal pneumatic muscle is calculated as $5.19 \mathrm{~N}$ and $15.2 \%$, respectively, from Eq. (6) and (7). The blocked forces are similar, but the strain prediction is much higher than $7.66 \%$ strain obtained from GFMs. It is usual to see smaller strains in under-inflated McKibben muscles. The theory is an ideal case and assumes that any internal pressure will generate the full strain predicted from Eq. (7). However, in real systems the braid friction and bladder's resistance to expansion mean that the strain is lower than ideal. Pneumatic McKibben muscles of a similar size to those used in this study typically generate strains of $30 \%$ and blocked forces of $100 \mathrm{~N}$ [30], however, these systems also use pressures of up to 500 $\mathrm{kPa}$ that are much larger than demonstrated here from gel swelling. 


\section{Conclusions}

A novel concept of hydrogel bead-filled braided muscle (GFM) has been demonstrated where the muscle was prepared by impregnating ionic-covalent entanglement hydrogel beads into the commercial braided sleeve. The beads are composed of alginate biopolymer and PNIPAAm, and the volume transition of the hydrogel beads was significant when the beads were equilibrated in water at temperatures above and below the LCST. A new actuation test method has also been introduced by connecting a tensile spring with the muscle in series. Fundamental characteristics (such as free stroke, blocked force, muscle stiffness, etc.) of linear muscles can be evaluated in a one-step process by using this method. A comprehensive study of enhancing muscle performance was conducted by altering the gel concentration, and the free strain and blocked force of the GFM have been found as high as $7.66 \%$ and $5.14 \mathrm{~N}$, respectively, when cooled at $8^{\circ} \mathrm{C}$ with complete reversibility when heated at $60^{\circ} \mathrm{C}$. Theoretical understanding of the blocked force generated from GFMs has been achieved by calculating the pressure generation of the swelling gel and further compared with the pressure expected from the volume increase of the gel beads. Reasonable agreement has been found in between the theoretical and experimental results and the analysis highlights the limited pressure available from the ICE gels used in the present study.

Response time, due to the diffusion properties of the hydrogel, still appears to be the limiting factor of the actuator design. However, the study on water-immersed actuation indicates that these muscles can be manipulated for constructing fluidic valve, hygroscopic smart textiles, and portable mass-lifter to be operated in aqueous media that is subjected to temperature fluctuation. It was also found that this muscle could be useful as a real-world actuator system without requiring preconditioning (training cycles) that is a noteworthy downside among other polymer artificial muscles. Further study to improve the response time such hydrogel based McKibben muscle is underway that considers different gel composition, gel geometry, braid construction, and braid geometry.

\section{Acknowledgements}

The authors thank the Australian Research Council Centre of Excellence for Electromaterials Science (ACES) for financial support and Ms. Jessica Grozdanov for assistance with sample preparation.

\section{References}

[1] Y. Bar-Cohen, Electroactive Polymer (EAP) Actuators as Artificial Muscles: Reality, Potential, and Challenges: SPIE Press; 2001.

[2] H. Cheng, Y. Hu, F. Zhao, Z. Dong, Y. Wang, N. Chen, et al., Moisture-Activated Torsional Graphene-Fiber Motor, Advanced Materials, 26(2014) 2909-13.
[3] C. Gonçalves, A. Ferreira da Silva, J. Gomes, R. Simoes, Wearable E-Textile Technologies: A Review on Sensors, Actuators and Control Elements, Inventions, 3(2018) 14.

[4] J. Guo, C. Xiang, T. Helps, M. Taghavi, J. Rossiter, Electroactive textile actuators for wearable and soft robots, 2018 IEEE International Conference on Soft Robotics (RoboSoft)2018, pp. 339-43.

[5] C.S. Haines, M.D. Lima, N. Li, G.M. Spinks, J. Foroughi, J.D.W. Madden, et al., Artificial Muscles from Fishing Line and Sewing Thread, Science, 343(2014) 868-72.

[6] A. Maziz, A. Concas, A. Khaldi, J. Stålhand, N.-K. Persson, E.W.H. Jager, Knitting and weaving artificial muscles, Science Advances, 3(2017) e1600327.

[7] E.W.H. Jager, O. Inganäs, I. Lundström, Microrobots for Micrometer-Size Objects in Aqueous Media: Potential Tools for Single-Cell Manipulation, Science, 288(2000) 2335-8.

[8] S. Shahidi, B. Moazzenchi, Carbon nanotube and its applications in textile industry - A review, The Journal of The Textile Institute, 109(2018) 1653-66.

[9] E. Smela, Conjugated Polymer Actuators for Biomedical Applications, Advanced Materials, 15(2003) 481-94.

[10] G.M. Spinks, Stretchable artificial muscles from coiled polymer fibers, Journal of Materials Research, 31(2016) 2917 27.

[11] J.D. Greer, T.K. Morimoto, A.M. Okamura, E.W. Hawkes, Series Pneumatic Artificial Muscles (sPAMs) and Application to a Soft Continuum Robot, IEEE Int Conf Robot Autom, 2017(2017) 5503-10.

[12] H.D. Yang, B.T. Greczek, A.T. Asbeck, Modeling and Analysis of a High-Displacement Pneumatic Artificial Muscle With Integrated Sensing, Frontiers in Robotics and AI, 5(2019).

[13] T. Akagi, S. Dohta, H. Kuno, M. Ihara, Improvement of McKibben Artificial Muscle with Long Stroke Motion and Its Application, Journal of System Design and Dynamics, 4(2010) 538-51.

[14] T. Nuchkrua, T. Leephakpreeda, Actuation of Pneumatic Artificial Muscle via Hydrogen Absorption/Desorption of Metal Hydride-LaNi5, Advances in Mechanical Engineering, 7(2015) 364306.

[15] T. Leephakpreeda, Mathematical Modeling of Pneumatic Artificial Muscle Actuation via Hydrogen Driving Metal Hydride-LaNi5, Journal of Bionic Engineering, 9(2012) 1108.

[16] R. Tiwari, M.A. Meller, K.B. Wajcs, C. Moses, I. Reveles, E. Garcia, Hydraulic artificial muscles, Journal of Intelligent Material Systems and Structures, 23(2012) 301-12. [17] D. Sangian, S. Naficy, G.M. Spinks, B. Tondu, The effect of geometry and material properties on the performance of a small hydraulic McKibben muscle system, Sensors and Actuators A: Physical, 234(2015) 150-7.

[18] R. Morita, H. Nabae, G. Endo, K. Suzumori, A proposal of a new rotational-compliant joint with oil-hydraulic McKibben artificial muscles, Advanced Robotics, 32(2018) 511-23.

[19] M. Meller, J. Chipka, A. Volkov, M. Bryant, E. Garcia, Improving actuation efficiency through variable recruitment hydraulic McKibben muscles: modeling, orderly recruitment 
control, and experiments, Bioinspiration \& Biomimetics, 11(2016) 065004.

[20] W. Michael, T.M. T., M. Yiğit, P. Yong-Lae, M. Annan, D. Ye, et al., Pneumatic Energy Sources for Autonomous and Wearable Soft Robotics, Soft Robotics, 1(2014) 263-74.

[21] B. Tondu, S. Mathé, R. Emirkhanian, Low pH-range control of McKibben polymeric artificial muscles, Sensors and Actuators A: Physical, 159(2010) 73-8.

[22] C. Santulli, S.I. Patel, G. Jeronimidis, F.J. Davis, G.R. Mitchell, Development of smart variable stiffness actuators using polymer hydrogels, Smart Materials and Structures, 14(2005) 434-40.

[23] J. Law, R. Rennie, A Dictionary of Physics: Oxford University Press; 2015.

[24] J. Shan, J. Chen, M. Nuopponen, H. Tenhu, Two Phase Transitions of Poly(N-isopropylacrylamide) Brushes Bound to Gold Nanoparticles, Langmuir, 20(2004) 4671-6.

[25] G. Gerlach, K.F. Arndt, Hydrogel Sensors and Actuators: Engineering and Technology: Springer Berlin Heidelberg; 2009.

[26] A. Chetty, J. Kovács, Z. Sulyok, A. Meszaros, J. Fekete, A. Domján, et al., A versatile characterization of poly $(\mathrm{N}-$ isopropylacrylamide-co-N,N'-methylene-bis-acrylamide) hydrogels for composition, mechanical strength, and rheology, Express Polymer Letters, 7(2012) 95-105.

[27] H. Omidian, S.-A. Hasherni, F. Askari, S. Nafisi, Swelling and Crosslink Density Measurements for Hydrogels, Iranian journal of polymer science \& technology, 3(1994)

[28] S.S. Ilić-Stojanović, L.B. Nikolić, V.D. Nikolić, J.R. Milić, S.D. Petrović, G.M. Nikolić, et al., Potential application of thermo-sensitive hydrogels for controlled release of phenacetin, Hemijska industrija, 66(2012) 831-9.

[29] F. Ganji, F.S. Vasheghani, F.E. VASHEGHANI, Theoretical description of hydrogel swelling: a review, (2010).

[30] B. Tondu, Modelling of the McKibben artificial muscle: A review, Journal of Intelligent Material Systems and Structures, 23(2012) 225-53.

[31] M. Biron, Mechanical Properties, in: M. Biron (Ed.) Material Selection for Thermoplastic Parts, William Andrew Publishing, Oxford, 2016, pp. 261-337.

[32] P. Voudouris, D. Florea, P. van der Schoot, H.M. Wyss, Micromechanics of temperature sensitive microgels: dip in the Poisson ratio near the LCST, Soft Matter, 9(2013) 7158-66. 